\title{
Mucosal Antibodies to Haemophilus influenzae Type b Capsular Polysaccharide
}

\author{
JANET R. GILSDORF AND W. MICHAEL MCDONNELL \\ Department of Pediatrics, C. S. Mott Children's Hospital and the University of Michigan Medical School, \\ Ann Arbor, Michigan 48109-0244
}

\begin{abstract}
Levels of salivary antibodies directed against Haemophilus influenzae b (Hib) capsule, measured by ELISA and standardized to the total salivary IgA, were compared among 57 patients with Hib infections, 117 household and 49 day care contacts of patients, and 53 control individuals with no known contact with Hib. Nineteen of the household or day care contacts were throat or nasopharyngeal culture positive for Hib. Eighty \% of evaluable patients had polyribosylribitol phosphate (PRP)specific IgA in their saliva, compared with $36 \%$ of contacts who were throat or nasopharyngeal culture positive for Hib, $53 \%$ of contacts who were throat or nasopharyngeal culture negative for $\mathrm{Hib}$, and $\mathbf{3 0 \%}$ of control children. In patients, no correlation between age and level of salivary anti-PRP antibody was seen, but patients less than 3 y old were more likely to have these antibodies than were older patients. Salivary PRP-specific IgA antibodies, associated with either Hib colonization or PRP vaccination, tended to decline over time. Thus, PRP-specific IgA antibodies can be identified in the saliva of children over a wide age range, including those colonized with Hib or vaccinated with PRP, but these antibodies appear to decline after antigenic stimulation ceases. (Pediatr Res 29: 420-423, 1991)
\end{abstract}

\section{Abbreviations}

Hib, Haemophilus influenzae, type b PBS-T, phosphate buffered saline with $0.3 \%$ Tween 20 PRP, polyribosylribitol phosphate

Hib is a major cause of serious pediatric infections, such as meningitis, facial cellulitis, pneumonia, septic arthritis, and epiglottitis, infecting approximately 20000 children in the United States annually (1). In children, protection against $\mathrm{Hib}$ infection has been correlated with the presence of serum antibodies directed against the capsular polysaccharide of $\mathrm{Hib}, \mathrm{PRP}$, and with serum bactericidal activity against $\operatorname{Hib}(2,3)$. The development of serum anti-PRP antibodies is age-related, inasmuch as young infants are unable to mount a sustained immune response in serum to this, as well as other, polysaccharide antigens $(4,5)$.

In addition to their presence in serum, antibodies against PRP have also been identified in mucosal secretions, such as saliva, nasal secretions, and breast milk (6-11). These mucosal antibodies, which are primarily IgA, are produced by mucosal plasma cells in response to mucosal colonization with $\mathrm{Hib}$ or other bacteria that possess cross-reacting antigens (12), as well as to

Received December 11, 1989; accepted December 18, 1990

Correspondence and reprint requests: Janet R. Gilsdorf, M.D., F2438 C.S. Mott Children's Hospital/0244, University of Michigan Medical Center, 1500 E. Medical Center Drive, Ann Arbor, MI 48109-0244.

Supported in part by Public Health Service Grants AI-20934-03 and 5M01-RR00042 from the National Institutes of Health. immunization with PRP $(7,8)$. The "common mucosal immune system," in which immunocompetent lymphoid cells migrate to multiple mucosal surfaces, allows antigenic stimulation at one mucosal site to result in the production of antigen-specific IgA antibodies at distant mucosal sites (13).

The focus of this study was to identify possible correlations between salivary PRP-specific IgA levels and various epidemiologic factors such as age, colonization status, and immunization status in patients with $\mathrm{Hib}$ infections and their contacts. In addition, we investigated the persistence of PRP-specific IgA antibodies in salivary secretions.

\section{MATERIALS AND METHODS}

Population surveyed. We measured the salivary antibodies to PRP in 57 patients, ages $6 \mathrm{wk}$ to $108 \mathrm{mo}$, hospitalized at C.S. Mott Children's Hospital with invasive Hib infections, including 36 with meningitis, eight with epiglottitis, three with bacteremia, two with arthritis, seven with cellulitis, and one with pneumonia (Table 1). None of these patients had received an Hib vaccine before illness. In addition, we studied 117 household contacts and 49 child care contacts of the patients. As a control group, we studied 53 individuals who had no known contact with Hib patients, including 46 children attending a control child care program. Three of the 57 patients $(5 \%)$ were black and one was Asian; the remainder were Caucasian. Nine of the 117 household contacts $(7.6 \%)$ were black and one was Asian. The predominance of Caucasians reflects the ethnic character of the population served by our medical center.

Informed consent was obtained from the subjects or their parents or guardians for obtaining the saliva samples. This study was approved by the Committee to Review Grants for Clinical Research and Investigation Involving Human Beings of the University of Michigan Medical Center.

Sample collection. Unstimulated salivary samples from adults and older children were collected directly into sterile polystyrene or polypropylene specimen containers. Salivary samples from babies were collected by aspirating oral secretions with a soft, plastic transfer pipette. Salivary samples were immediately placed on ice for transfer to the laboratory and then frozen at $70^{\circ} \mathrm{C}$ until the antibody assay was performed. Before assay, the samples were defrosted and centrifuged at $1000 \times g$ for $5 \mathrm{~min}$ to remove cells and debris. Specimens from patients and from household contacts were obtained within $7 \mathrm{~d}$ of diagnosis of the patient's $\mathrm{Hib}$ infection. Specimens from day care contacts were obtained within 1 mo of diagnosis of the day care child with Hib infection. A subset of patients, as well as contacts shown to be colonized with $\mathrm{Hib}$, provided repeat salivary samples collected at varying time intervals for up to 14 mo after the first specimen. Throat or nasopharyngeal culture specimens were obtained from all participants at the time of saliva collection and processed on selective chocolate agar (14) for the isolation of Hib, identified using standard procedures (15).

Salivary PRP-specific IgA assays. Salivary IgA directed against 
PRP was measured using an ELISA as previously described (16). Wells of polystyrene microtiter plates (NUNC-Intermed Vangard Int'l Inc., Neptune, NJ) were coated with PRP, which was isolated from Hib strain Eagan (17) and then conjugated to tyramine (18), and incubated for $1 \mathrm{~h}$ with saliva diluted $1: 20$ in PBS-T. The reaction was then developed with affinity-purified goat anti-human secretory IgA conjugated to horseradish peroxidase (Cappel Laboratories, Malvern, PA) for $1 \mathrm{~h}$. The enzyme substrate O-phenylenediamine (Sigma Chemical Co., St. Louis, MO) was added and, after termination of the reaction with sulfuric acid, the OD of the contents of the wells was measured on a Titertek multi-scan spectrophotometer (Flow Laboratories, Rockville, MD) at $492 \mathrm{~nm}$. Negative control wells contained either diluent without saliva, or saliva obtained from an agammaglobulinemic patient who had no detectable salivary IgA. Positive control wells were included on every plate and contained saliva from a laboratory worker with high levels of PRP-specific IgA. To document that this assay was measuring PRP specifically, we tested a subset of positive control saliva before and after adsorption with purified PRP. This adsorption procedure removed the reactivity from the positive saliva.

To standardize test values for potential dilutional differences between saliva samples (19), we chose to express these values as PRP-specific IgA per mg total IgA in the saliva sample (6-10). Measurement of total IgA in the secretory specimens was performed using an antigen capture ELISA. The microtiter plates were coated with $\alpha$-chain-specific, affinity-purified goat antihuman IgA (Tago, Burlingame, CA), and saliva specimens, diluted 1:3200 in PBS-T, were added. The reaction was then developed with $\alpha$-chain-specific, affinity-purified goat anti-human IgA conjugated to horseradish peroxidase (Tago) diluted $1: 4000$ in PBS- $T$. The enzyme substrate O-phenylenediamine (Sigma Chemical Co.) was added, and the reaction was terminated using sulfuric acid. The OD values of the wells were determined on a Titertek multi-scan spectrophotometer at 492 $\mathrm{nm}$. Included in each assay were a negative control saliva from an agammaglobulinemic patient that contained no IgA and a positive control saliva shown by nephelometry using a serum Ig standard reference, QM-2, control A (Kallestad, August, TX), to contain $14.3 \mathrm{mg} \mathrm{IgA} / \mathrm{dL}$. The lower limit of detection in this assay ranged from 0.5 to $4.4 \mathrm{mg} / \mathrm{dL}$ total IgA.

Expression of anti-PRP-specific IgA antibody levels. Because of day-to-day differences in the OD of the negative and positive control samples [approximately $15 \%$ (16)], the OD values of the test specimens were normalized using the following formula (20):

$$
\frac{\text { OD test sample }- \text { OD negative control }}{\text { OD positive control - OD negative control }} \times 100
$$

Table 1. Demographic characteristics of patients, contacts, and control individuals

\begin{tabular}{|c|c|c|c|}
\hline & $n$ & $\begin{array}{l}\text { Aean age (range } \\
\text { (mo) }\end{array}$ & $\%$ Female \\
\hline Patients & 57 & $25.2(2-108)$ & 50.8 \\
\hline Meningitis & 36 & $22.4(2-108)$ & 47 \\
\hline Epiglottitis & 8 & $34.7(19-48)$ & 62.5 \\
\hline Bacteremia & 3 & $25.0(2-60)$ & 33 \\
\hline Arthritis & 2 & $10.5(9-12)$ & 50 \\
\hline Cellulitis & 7 & $15.0(7-40)$ & 71.4 \\
\hline Pneumonia & 1 & 3 & \\
\hline Household contacts & 117 & & 51 \\
\hline Adults & 76 & & 51 \\
\hline Children & 41 & $76.8(5-192)$ & \\
\hline Child care contacts & 49 & & 69 \\
\hline Adults & 11 & & \\
\hline Children & 38 & $38.7(5-120)$ & \\
\hline No contacts & 53 & & 34 \\
\hline Adults & 5 & & \\
\hline Children & 48 & $54(6-72)$ & \\
\hline
\end{tabular}

The normalized test value was expressed as ELISA units. For each sample, to control for concentration differences of IgA from sample to sample, the normalized OD value for the PRP-specific IgA was divided by the normalized value for the total IgA and the results expressed as PRP-specific IgA per total $\operatorname{IgA}(7)$. The lower limit of detection of PRP-specific IgA per total IgA in ELISA units was 2 SD above the negative control.

Analysis of data. Statistical analysis, which was performed using the CLINFO program (Bolt, Beranek, and Newman, Inc., Boston, MA) of the University of Michigan Clinical Research Center, included tests of correlation using Pearson's coefficient of correlation and inferences on proportions using $\chi^{2}$ analysis and Fisher's exact test.

\section{RESULTS}

Saliva specimens collected from 57 patients with cultureproven $\mathrm{Hib}$ infections were available for study. All 57 patients were throat or nasopharyngeal culture negative for $\mathrm{Hib}$ at the time the saliva specimen was collected. Of the 166 household or child care contacts of the patients, 147 were throat or nasopharyngeal culture negative for $\mathrm{Hib}$ and 19 (10 household and nine child care contacts) were asymptomatic Hib carriers. All 53 noncontact (control) subjects were throat or nasopharyngeal culture negative for Hib. Among the 337 saliva samples tested, $101(29.9 \%)$ lacked detectable IgA, so PRP-specific IgA values standardized for total IgA could not be quantitated. Fifty-one \% of samples from patients were negative for total IgA compared with $29.9 \%$ of all samples $(p=0.001)$. Furthermore, $70 \%$ of samples obtained from children under 12 mo of age (the majority were patients) and $60.9 \%$ of samples obtained from children under 24 mo of age were negative for total $\operatorname{IgA}(p=0.00001)$. Nine of 29 samples (31\%) from patients over age 4 mo were negative for salivary total $\operatorname{IgA}(p=0.005)$, suggesting that low total IgA was related to young age rather than to patient status. The absence of detectable IgA did not correlate with length of specimen storage.

Table 2 documents the relationship of salivary PRP-specific IgA to Hib colonization. Patients with Hib infection were more likely to have PRP-specific IgA in their saliva (sampled within 7 $\mathrm{d}$ of diagnosis) than the other groups tested, including culturepositive contacts, nonvaccinated culture-negative contacts, and nonvaccinated, noncontact (control) subjects. Among patients, children $<3$ y of age were more likely to have salivary PRPspecific IgA [(21 of $23(91.3 \%)]$ compared with patients $>3$ y [four of eight $(50 \%) ; p=0.001$ ] . This age differential was not seen among culture negative contacts; three of nine culture negative contacts $(33 \%)<3$ y old had salivary PRP-specific IgA compared with 72 of $133(54 \%)>3$ y old $(p=0.33)$. Too few culture-positive contacts or noncontact (control) individuals $<3$ y old were studied to make valid comparisons.

The relationship between age and salivary PRP-specific antibody levels in patients is depicted in Figure 1, and no correlation was seen $(r=-0.077)$. Eight of 10 patients under age $2 \mathrm{y}$, and

Table 2. Presence of PRP-IgA in patients, culture-positive and nonvaccinated culture-negative contacts, and nonvaccinated control individuals

\begin{tabular}{lrc}
\hline & $n$ & No. with PRP-IgA (\%) \\
\hline Patients & 31 & $25(80)^{*}$ \\
Meningitis & 16 & $14(87)$ \\
Epiglottitis & 6 & $3(50)$ \\
Cellulitis & 3 & $3(100)$ \\
Other & 6 & $5(83)$ \\
Culture-positive contacts & 14 & $5(36)^{*} \dagger$ \\
Culture-negative contacts & 125 & $68(54) \dagger$ \\
Controls & 20 & $6(30) \dagger$ \\
\hline
\end{tabular}

$* \chi^{2}=8.761, d f=1, p=0.003$

$\dagger \chi^{2}=5.304, d f=2, p=0.07$. 


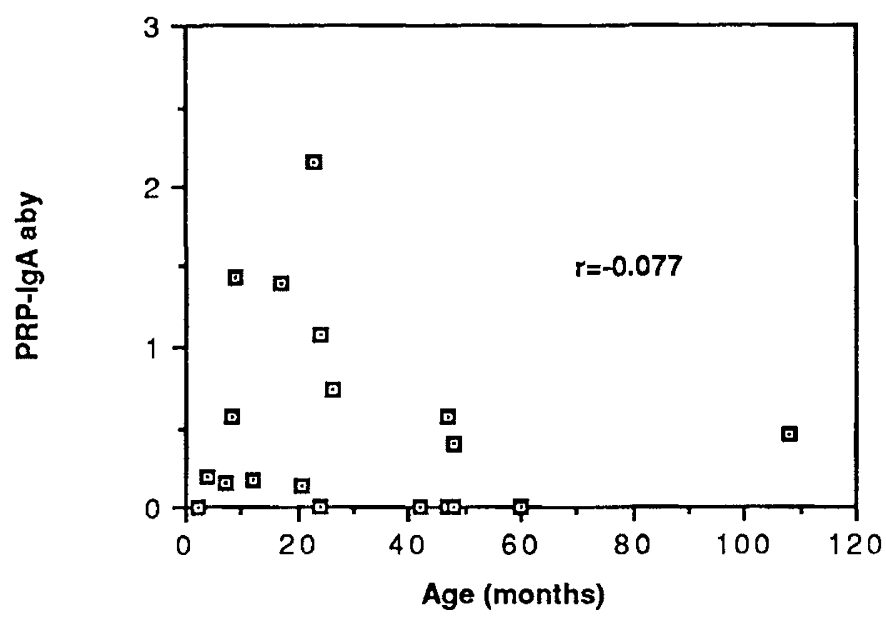

Fig. 1. Relationship of age to salivary anti-PRP antibody levels (expressed as ELISA units of PRP antibody/mg total $\operatorname{IgA}$ ) in patients with Hib infections. Data shown are for samples obtained from patients within $7 \mathrm{~d}$ of hospitalization.

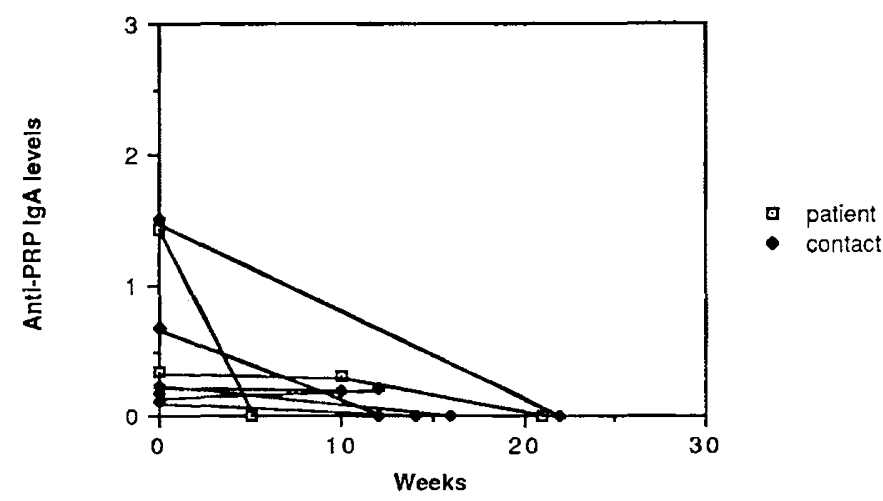

Fig. 2. Duration of salivary PRP-IgA antibodies (expressed as ELISA units of PRP antibody/mg total IgA) among patients and asymptomatic carriers.

five of six patients under age 1 y possessed salivary PRP-specific IgA. None of these patients were throat or nasopharyngeal culture positive for Hib at the time the salivary specimens were collected.

The presence of PRP-specific antibodies in saliva specimens over time was determined for a subset of patients and contacts who were followed for as long as $14 \mathrm{mo}$. Salivary PRP-specific IgA antibodies persisted in three of six individuals followed for 6-8 wk, in two of four individuals followed for 3-4 mo, and in two of three individuals followed for 5-6 mo. Thus, the salivary PRP-specific IgA was detected only in seven of 13 individuals 2 to 6 mo beyond the time of initial detection. Among the individuals who were initially antibody negative, again none of the four patients or carriers subsequently possessed antibody, whereas six of the eight culture-negative contacts had developed antibody. This suggests that, during the follow-up period, some of the culture-negative contacts may have transiently acquired the organism and developed mucosal PRP-specific antibody.

Figure 2 shows the levels of salivary PRP-specific IgA over time. Within approximately 20 wk from the time of documentation of the presence of antibody, none of the patients showed an increase in antibody level, and the trend was distinctly toward disappearance of antibody.

Salivary PRP-specific IgA antibody levels were measured in children who were child care contacts of patients and who attended day care facilities that were heavily $(>33.3 \%$ of children were culture positive) or lightly ( $<33.3 \%$ of children were culture positive) colonized with Hib. These levels were compared with those of noncontact children attending a control child care center in which none of the children had a positive respiratory culture for Hib. Six of 15 children (40\%) in heavily colonized day care centers, two of six children (33\%) in a lightly colonized day care center, and 10 of 27 noncontact (control) children $(37 \%)$ possessed salivary PRP-specific IgA. These differences were not significant $\left(\chi^{2}=0.09, d f=2, p=0.9\right)$.

Some of the children attending the control day care center had received the Hib vaccine and were excluded from analyses described above. However, from these vaccinated children, we were able to compare the effect of immunization on salivary PRP. specific IgA antibodies. Five of the eight immunized children $(62.5 \%)$, ranging in age from 32 to $72 \mathrm{mo}$, possessed antibody, compared with five of 19 nonimmunized children (26.3\%), also ranging in age from 32 to $72 \mathrm{mo}$ ( $p=0.102$, Fisher's exact test). In addition, we correlated the level of PRP-specific IgA antibodies with time since immunization, ranging from 2.5 to $12.5 \mathrm{mo}$. A strong negative correlation $(r=-0.81, p=<0.05)$ was seen between PRP-specific IgA level and time since immunization, again suggesting a decline in salivary anti-PRP antibody level over time.

\section{DISCUSSION}

The presence of anti-PRP IgA antibodies has been documented in several different mucosal secretions, including breast milk, nasal secretions, and saliva (6-11), and the levels of these antibodies in saliva have correlated with levels in nasal mucus (10). Mucosal anti-PRP-IgA antibodies are thought to be naturally occurring in adults $(9,10)$ and, in children, have been documented as a response to Hib infection (6). After immunization with PRP vaccine, both adults and children showed a rise in the level of PRP-specific IgA antibodies in secretions (7, 8).

Our study confirms the presence of PRP-specific IgA antibodies in the saliva of children, and compares the presence and levels of these antibodies among patients with Hib infection, contacts of patients, and normal controls. PRP-specific IgA was more frequently detected in saliva from patients than in saliva from culture-positive contacts, culture-negative contacts, and noncontact (control) subjects. Although the pharyngeal lymphoid tissues of both culture-positive contacts and patients are exposed to PRP antigen during nasopharyngeal colonization with $\mathrm{Hib}$, the patients may have had a higher bacterial concentration and, thus, a stronger antigenic stimulus. Similarly, mucosal antibody production in patients may be enhanced by an additional factor contributing to mucosal bacterial invasion, such as viral disruption of the mucosa. Alternatively, the bacteria in the pharynges of culture-positive contacts may have bound to the mucosal antibodies so that we were not able to detect their presence (19).

In addition to documenting the presence of PRP-specific IgA antibodies in saliva, our studies explored the persistence of these antibodies. We found that levels of mucosal IgA antibodies against Hib PRP declined after cessation of mucosal exposure, which is similar to findings with mucosal antibodies against Vibrio cholera (21), Neisseria gonhorrhoeae (22), and Streptococcus mutans (23). Although persistence of mucosal antibodies to Haemophilus influenzae has not previously been measured by antibody assay, Clancy et al. (24) documented protection against acute bronchitis in adults after oral immunization with formalin killed $H$. influenzae (type not specified); the protection was observed for 3 mo after administration of the vaccine, but was not apparent 1 y later.

A number of studies have shown that, after either invasive Hib infection or vaccination with the type $b$ capsular polysaccharide PRP, serum anti-PRP antibodies cannot be demonstrated in young infants. This age restriction in the development of antipolysaccharide antibodies, however, might not exist with IgA mucosal antibodies. We demonstrated anti-PRP IgA antibodies in four of five children younger than 6 mo of age $(1,2,4$, and 5 mo). These results confirm those of other investigators $(6,11)$, who have also shown that infants under 1 y of age can generate PRP-specific IgA in mucosal secretions, even in the absence of 
PRP-specific IgG in serum. Rosales et al. (11) attribute the dissociation of the presence of anti-PRP antibody in serum and saliva to "oral tolerance." They postulate that bacterial colonization of mucosal surfaces suppresses the development of circulating antibody against the colonizing antigens, possibly by selective activation of mucosal suppressor $T$ cells that migrate to the spleen and suppress production of $\operatorname{IgG}$ or $\operatorname{IgM}$, but not $\operatorname{IgA}$ antigen-specific antibodies (25). Because oral tolerance appears to be limited to infants, the postulated suppression would need to be a developmental phenomenon that disappears with maturation. Alternatively, the production of antipolysaccharide antibodies within the mucosal lymphoid tissue may be regulated by a mechanism separate from that of systemic lymphoid tissue.

The function of mucosal PRP-specific IgA antibodies in host defense is unclear. Mucosal IgA has been postulated to aggregate bacteria and facilitate clearance by the mucociliary blanket, to inhibit microbial adherence and colonization, and to neutralize viruses and bacterial toxins (25). IgA from the bronchial secretions of patients with nontypable $H$. influenzae pneumonia was shown to block the bactericidal and opsonizing activity of serum antibody to nontypable $H$. influenzae (26). In addition, the ability of anti-PRP IgA antibodies in serum to protect against Hib infection is unclear, inasmuch as they are neither bactericidal nor opsonic and, in passive protection experiments, did not protect rats against $\mathrm{Hib}$ challenge (27).

Thus, mucosal anti-PRP antibodies may differ significantly from circulating anti-PRP antibodies. Mucosal colonization can stimulate production of mucosal anti-PRP antibodies in very young children, but these antibodies decline after antigenic stimulation ceases, irrespective of age.

Acknowledgment. The authors thank Steven Schmaltz from the University of Michigan Clinical Research Center for his assistance in statistical analysis.

\section{REFERENCES}

1. ACIP (Advisory Committee Immunization Practices) 1985 Polysaccharide vaccine for prevention of Haemophilus influenzae type b disease. MMWR 34:201-205

2. Käyhty H, Peltöla H, Karanko V, Mäkelä PH 1983 The protective level of serum antibodies to the capsular polysaccharide of Haemophilus influenzae type b. J Infect Dis 147:1100

3. Fothergill LD, Wright $J 1933$ Influenzal meningitis: the relation of age incidence to the bactericidal power of blood against the causal organism. J Immunol 24:273-284

4. Smith DH, Peter G, Ingram DL, Harding AL, and Anderson P 1973 Responses of children immunized with capsular polysaccharide of Haemophilus influenzae, type b. Pediatrics 52:637-644

5. Käyhty $\mathrm{H}$, Jousimies-Somer $\mathrm{H}$, Peltöla $\mathrm{H}$, Mäkelä $\mathrm{PH} 1981$ Antibody response to capsular polysaccharides of groups A and C Neisseria meningitidis and Haemophilus influenzae type $\mathrm{b}$ during bacteremic disease. J Infect Dis 143:32-36

6. Pichichero ME. Hall $\mathrm{CB}$, Insel RA $1981 \mathrm{~A}$ mucosal antibody response following systemic Haemophilus influenzae type b infection in children. J Clin Invest 67:1482-1489

7. Insel RA, Amstey M, Pichichero ME 1985 Postimmunization antibody to the Haemophilus influenzae type b capsule in breast milk. J Infect Dis 152:407408

8. Pichichero ME, Insel RA 1983 Mucosal antibody response to parenteral vaccination with Haemophilus influenzae type b capsule. J Allergy Clin Immunol 72:481-486

9. Pichichero ME Sommerfelt AE Steinhoff MC Insel RA 1980 Breast milk antibody to the capsular polysaccharide of Haemophilus influenzae type b. J Infect Dis 142:694-698

10. Pichichero ME, Insel RA 1982 Relationship between naturally occurring human mucosal and serum antibody to the capsular polysaccharide of Haemophilus influenzae type b. J Infect Dis 146:243-248

11. Rosales SV, Lascolea LJ, Ogra PL 1984 Development of respiratory mucosal tolerance during Haemophilus influenzae type $b$ infection in infancy. $J$ Immunol 132:1517-1521

12. Mestecky J, McGhee JR, Arnold RR, Michalek SM, Prince SJ, Babb JL 1978 Selective induction of an immune response in human external secretions by ingestion of bacterial antigen. $\mathrm{J}$ Clin Invest 61:731-787

13. McNabb PC, Tomasi TB 1981 Host defense mechanisms at mucosal surfaces. Annu Rev Microbiol 35:477-563

14. Chapin KC, Doern GV 1983 Haemophilus influenzae from specimens contaminated with upper respiratory tract microbial flora. J Clin Microbiol 17:11631165

15. Kilian M 1980 Haemophilus. In: Lennette EH (ed) Manual of Clinical Microbiology. American Society for Microbiology. Washington, DC, pp 330-336

16. McDonnell WM, Gilsdorf JR, Kent JB, Sheagren JN 1988 Relationship of health-related variables to levels of antipolyribosylribitol phosphate antibodies in adults. J Clin Microbiol 26:1202-1206

17. Kuo JS, Monju RS, Schwalbe RS, McCoy DW 1981 A radioactive antigenbinding assay for the measurement of antibody to Haemophilus influenzac type b capsular polysaccharide. J Immunol Methods 43:35-47

18. Antohyn BF Concepcion NF, McGeary SA, Ward JI, Heiner DC, Shapshak $P$, Insel RA 1982 Immunospecificity and quantitation of an enzyme-linked immunosorbent assay for group B streptococcal antibody. J Clin Microbiol $16: 350-354$

19. Gahnberg L, Krasse B 1981 Salivary immunoglobulin A antibodies reacting with antigens from oral streptococci: longitudinal study in humans. Infect Immun 33:697-703

20. Shale DJ, Faux JA 1985 The evaluation of a quantitative enzyme-linked immunosorbent assay (ELISA) for anti-Aspergillus fumigatus IgG. J Immunol Methods 77:197-205

21. Svennerhom AM, Jertborn M, Gothefors I Karim AMMM, Sack DA, Holmgren J 1984 Mucosal antitoxic and antibacterial immunity after cholera disease and after immunization with a combined $b$ subunit-whole cell vaccine. J Infect Dis 149:884-893

22. O'Reilly RJ, Lee L, Welch BG 1976 Secretory IgA antibody responses to Neisseria gonorrhoeae in the genital secretions of infected females. $J$ Infect Dis 133:113-118

23. Gregory RL, Filler SJ 1987 Protective secretory immunoglobulin A antibodies in humans following oral immunization with Streptococcus mutans. Infect Immun 55:2409-2415

24. Clancy R, Murree-Allen K, Engel M, Cripps A, Yeung S 1985 Oral immunisation with killed Haemophilus influenzae for protection against acute bronchitis in chronic obstructive lung disease. Lancet 2:1395-1397

25. Bergmann KC. Waldman RH 1988 Stimulation of secretory antibody following oral administration of antigen. Rev Infect Dis 10:939-950

26. Musher DM, Goree A. Baughn RE, Birdsall HH 1984 Immunoglobulin A from bronchopulmonary secretions blocks bactericidal and opsonizing effects of antibody to nontypable Haemophilus influenzae. Infect Immun 45:36-40

27. Schreiber JR, Barrus V, Cates KL, Siber GR 1986 Functional characterization of human IgG, IgM, and IgA antibody directed to the capsule of Haemophilus influenzae type b. J Infect Dis 153:8-16 